\title{
APPETITI COME ENERGIE DISPERSE SECONDO LA "SALITA AL MONTE CARMELO" DI SAN GIOVANNI DELLA CROCE
}

"L’uomo non può vivere senza amore. Egli rimane per sé stesso un essere incomprensibile, la sua vita è priva di senso, se non gli viene rivelato l'amore, se non s'incontra con l'amore, se non lo sperimenta e non lo fa proprio, se non vi partecipa vivamente" ${ }^{1}$. Queste parole di san Giovanni Paolo II racchiuse nell'enciclica Redemptor hominis mostrano l'importanza capitale dell'amore nella vita umana. Solo esso dà significato a tutta la vita dell'uomo, la quale senza amore perde il senso e diventa incomprensibile. Questo amore ci è "rivelato" e deve essere "incontrato" e "partecipato vivamente". Dio stesso è questo amore (cf. $1 \mathrm{Gv} 4,8$ ) e lo si deve incontrare personalmente. Questo messaggio fondamentale del cristianesimo è stato vissuto e descritto in modo particolare da San Giovanni della Croce, chiamato spesso "Dottore dell'amore divino", una persona che affascina da molto tempo. Proclamato Dottore della Chiesa e riconosciuto come grande maestro della vita spirituale trova un interesse generale, anche ai nostri tempi.

Secondo san Giovanni della Croce in questa dinamica dell'amore entra strettamente una realtà appartenente alla natura stessa dell'uomo, descritta da s. Giovanni sotto la nozione degli appetiti (los apetitos). Questo termine viene usato da san Giovanni della Croce in tutti i suoi scritti. Esso viene usato dal Santo ben 579 volte fin dalla prima frase del primo libro della Salita al Monte Carmelo ${ }^{2}$.

Seguendo il pensiero di san Giovanni della Croce su questo tema, presentato specialmente nella Salita al Monte Carmelo, in questo articolo verrà precisata la nozione degli appetiti, la loro descrizione dal punto di vista psicologico e la

1 Giovanni PaOlo II, Redemptor hominis, n. 10.

2 Cfr. J.L. Astigarraga, A. Borrell, F.J.M. de Lucas, Concordancias de los escritos de san Juan de la Cruz, Roma 1990, p. 2075. 
loro valutazione teologale e morale. Infine vedremo quali sono le prime cure per la purificazione degli appetiti. Vedremo anche come tutta questa dinamica legata agli appetiti e il processo della loro purificazione sia collegato alla dinamica dell'amore e dell'unione con Dio, che sono la vocazione fondamentale dell'umo.

\section{NozIONE DEGLI APPETITI}

Ai tempi di san Giovanni della Croce il temine "appetiti" (apetitos) veniva usato raramente tranne che nella filosofia e nella teologia ${ }^{3}$. Nella teologia scolastica significava propriamente l'inclinazione psicologica verso un bene conosciuto ${ }^{4}$. Poiché la conoscenza umana è duplice (razionale e sensitiva), anche l'appetito si distingue in razionale e sensitivo ${ }^{5}$. Il primo si identifica con la volontà, il secondo è descritto come potenza organica radicata nell'anima che persegue il bene esterno e rifugge da ciò che è male secondo l'apprensione dei sensi esterni (vista, udito, ecc.), della estimativa e della fantasia (due sensi interni), interessando indirettamente la volontà e dipendendo da essa ${ }^{6}$.

Il nostro Autore, in genere, accoglie il pensiero scolastico sugli appetiti e lo applica alla vita spirituale. È da notare, però, che egli arricchisce molto il significato di tale nozione trattando gli appetiti non tanto "in astratto" ma come un "fenomeno reale", sperimentale, esprimente una realtà molteplice, e analizzandolo nelle sue diverse dimensioni: psicologica, teologica e morale. Benché egli ne parli continuamente, non li definisce con precisione, proprio perché non lo interessano tanto le distinzioni scolastiche quanto far capire che gli appetiti abbracciano praticamente l'uomo intero, nelle sue dimensioni e facoltà.

Qualche orientamento sul significato di questa nozione intesa nel senso sangiovanneo lo troviamo nell'affermazione di V. Capánaga:

Los apetitos humanos siguen el conocimiento sensible y racional, y reciben diversos nombres [... ]. San Juan de la Cruz habla de aficiones, asimiento, codicia, deseo de apetitos, ansia y pena, aprieto y ansia de amor, impetu y fuerza de pasión, osadia de amor, vehemencia y fuerza de amor, conato y fuerza, apasionarse en alguna afección, poner el corazón en una cosa, enamorarse de algo, apacentarse y detenerse en extraños gustos, apetecer y pedir, asirse a una cosa por afición, tener sed de algo, penar en deseo y ansia, apetecer y codiciar impacientemente, sentir ansia inflamada, inflamación y estimulo...

Cfr. F. Ruiz, S. Giovanni della Croce. Il santo, gli scritti, il sistema, Roma 1973, p. 162.

Cfr. S. Thomas, Summa theologiae, Alba-Romae $1962^{8}$, I, q. 80, a. 1.

Cfr. ibid. I, q. 80, a. 2.

6 Cfr. G.G. Pesenti, Appetito, in: Dizionario Enciclopedico di Spiritualità, a cura di E. Ancilli e del Pontificio Istituto di Spiritualità del Teresianum, Roma 1992², vol. I, p. 197. 
¿En qué consiste la esencia de un apetito o del apetito en general? En ir hacia algo que le falta. Su movimiento o tendencia 'nace del hambre y sed que tiene de lo que le falta' (N II,13,9)

Questa tendenza è legata ad ogni facoltà e al suo funzionamento che, in modo naturale e spontaneo, esprime l'essere di questa facoltà. Così la vista prova gusto nel guardare, l'udito nell'ascoltare, l'immaginazione nel fantasticare, l'intelletto nel discorrere e speculare, ecc. In questo senso tale tendenza è sicuramente giusta e perfino necessaria per poter operare.

Ciò che preoccupa Giovanni della Croce sono le facoltà affettive che, per loro stessa natura, sono già una tendenza verso un oggetto che le attira, considerato da loro come buono. In pratica si possono vedere qui due modi di creazione degli oggetti di interesse per le facoltà affettive: o la volontà sceglie un oggetto conveniente e si fa accompagnare dall'appetito e dalla passione, o viceversa - si lascia dominare dall'istinto e dalla passione ${ }^{8}$.

Nel primo caso la condizione è molto positiva: la forza della volontà aumenta perché "anche [le passioni] l'aiutano a sentire amore appassionante" (N II, 13,3). Quindi la passione dà una nuova forza e vigore alla volontà. Questa constatazione sta nella profonda sintonia con la psicologia contemporanea che malvolentieri si riferisce al concetto della "volontà pura", spogliata da ogni appoggio affettivo.

Molto diverso è il secondo caso. La volontà esiste e può fare molti sforzi e sacrifici ma sempre con lo scopo di soddisfare la passione e l'appetito di cui è schiava. Benché la volontà qui sia forte, è posta al servizio di qualcosa di inferiore e di indegno rispetto alle proprie capacità.

Bisogna notare come nell'uomo reale e storico questo secondo caso sia "normale" a causa del peccato originale. La teologia del peccato originale, elaborata dal Concilio di Trento, quindi praticamente ai tempi del nostro Santo, era molto ben conosciuta da lui e questo fattore dell'antropologia teologica è molto importante per il nostro Autore per descrivere e per valutare il fenomeno degli appetiti.

Secondo i dati biblici e quelli teologici l'uomo è stato creato da Dio per la comunione con Lui fin dal paradiso terrestre (cfr. Gen 2-3). Essendo in comunione d'amore con Dio, l'uomo era anche in armonia con sé stesso, era quindi interiormente integrato. La sua dimensione spirituale, unita attraverso l'amore con Dio ed a Lui sottomessa, integrava anche la sua dimensione sensitiva, così che anch'essa poteva essere unita a $\mathrm{Dio}^{9}$. L'uomo poteva quindi veramente

\footnotetext{
V. Capanága, San Juan de la Cruz. Valor psicológico de su doctrina, Madrid 1950, p. 158.

Cfr. F. Ruiz Salvador, S. Giovanni della Croce. Il santo, p. 668.

Cfr. Eulogio de la V. del Carmen, Antropologia sanjuanística, in: "Monte Carmelo", 69 (Madrid 1961), p. 85.
} 
amare Dio con tutto il cuore, con tutta l'anima e con tutte le forze (cfr. Dt 6,5). Essendo integrato in sé stesso l'uomo era anche integrato con il mondo esteriore: nelle relazioni interpersonali (p.e. l'unità e l'armonia tra Adamo ed Eva prima del peccato originale) e nell'uso delle creature che contemplava come qualcosa di meraviglioso e di una bellezza indicibile (cfr. C 4,3: 5,1-4). Esse, "piantate dalla mano dell'Amato" $(\mathrm{C} 4)^{10}$, conducevano in modo naturale l'anima verso Dio, narrando di Lui mille grazie (cfr. C 7).

In questa concretezza, realtà e storicità dell'uomo entra l'evento del peccato originale che profondamente cambia la verità sull'uomo. Il peccato originale ha rotto in tutte le dimensioni l'iniziale integrazione dell'esistenza umana. Anzitutto, il peccato, come atto di ribellione e disobbedienza a Dio, ha introdotto "'inimicizia tra l'uomo e Dio" (C 23,2), rompendo quell'armonia dell'amore che esisteva prima tra loro. Un'altra conseguenza di tale peccato è la perdita dell'integrazione dell'uomo con sé stesso e - come risultato - la disarmonia nelle sue potenze. "La natura umana [...] fu corrotta e perduta da Adamo per mezzo dell'albero proibito" (C 23,2). S. Giovanni ricorda nel primo libro della Salita che "l'anima dopo il peccato originale, è come prigioniera nel corpo mortale, soggetta alle passioni e agli appetiti naturali" (S I, 15,1). Quindi, pur essendo ontologicamente buona, la natura umana secondo il peso dei suoi appetiti dopo il peccato originale è "normalmente" sregolata. Infine, il peccato originale ha distrutto l'integrazione con il mondo esteriore. L'universo, tutte le creature, anche se buone in sé stesse, appaiono all'uomo così come egli stesso è; allora gli appetiti dell'uomo e la visione delle cose che essi implicano sono ugualmente impuri.

Queste conseguenze dannose della disintegrazione provocata dal peccato fanno parte della natura umana nella nostra situazione esistenziale e vengono comunemente sperimentate. Così, gli ostacoli nel cammino dell'amore hanno il loro fondamento nel disordine degli appetiti e nella disintegrazione interiore della persona umana.

10 In questo articolo saranno usate le seguenti abbreviazioni delle opere di s. Giovanni della Croce, riferite all'edizione italiana (S. Grovanni della Croce, Opere, a cura di Ferdinando di S. Maria, Roma 19916): AA - Altri avvisi (compresi in Parole di luce e d'amore); $\mathrm{C}$ - Cantico spirituale, seconda redazione (B); $\mathrm{N}$ - Notte oscura [ seguono: numero del libro (romano), capitolo, paragrafo]; S - Salita al Monte Carmelo [seguono: numero del libro (romano), capitolo, paragrafo (tit. - significa che la citazione si trova nel titolo del capitolo) ]; SA - Spunti di amore (compresi in Parole di luce e d'amore). 


\section{DesCrizione PSICOlOGiCA}

Vogliamo dare all'inizio una descrizione psicologica degli appetiti, anche se Giovanni della Croce comincia piuttosto con la loro valutazione teologale (cfr. $\mathrm{S} \mathrm{I}, 4,1-6,4)$. Ci sembra più naturale vedere prima come si manifesta la loro presenza nell'anima, cosa che può essere sperimentata fenomenologicamente da ciascuno di noi. Il Santo la descrive in modo molto suggestivo e pittoresco.

Giovanni della Croce come scrittore spirituale si interessa degli appetiti in quanto legati alla relazione dell'uomo con Dio. Siccome si rende conto dalla sregolatezza della natura umana dopo il peccato originale nell'ambito degli appetiti, san Giovanni nota la loro dannosità, perciò nei suoi scritti, specialmente nella Salita al Monte Carmelo, impiega questa espressione nella maggior parte in senso negativo (cfr. S I, 1,1; S I,4-5).

Il carattere negativo degli appetiti, sperimentati a livello psicologico, consiste soprattutto nei danni causati all'uomo. Nella Salita del Monte Carmelo egli elenca i cinque danni principali: stanchezza, tormento, oscurità, sudiciume e indebolimento dell'anima (cfr. S I,6,5).

Gli appetiti stancano l'anima, poiché essa si stanca e si affatica molto per conseguire ciò che le chiedono. Anche se l'uomo raggiunge quanto questi bramano, si stancherà ugualmente senza mai trovare soddisfazione, perché "è come un malato di febbre, che non sta bene finché questa non lo lascia libero" (S I,6,6).

Un altro danno è il tormento dell'anima, "come è tormentato uno schiavo incatenato, il quale non si riposa finché non è disciolto" (S I,7,1). Il grado del tormento dipende dall'intensità dell'appetito e dalla quantità degli appetiti ai quali si cede (cfr. S I,7,2).

Gli appetiti insudiciano l'anima. Le creature per un "uomo reale" sono come pece, che macchia colui che la tocca, cioè chi "appaga l'appetito della sua volontà in qualche creatura" (S I,9,1). L'anima che arde dal desiderio di qualche cosa, proprio a causa del calore di questo desiderio, rimane immonda e macchiata (cfr. ibid).

L'anima dominata dagli appetiti è indebolita, cosicché non ha più forza per progredire nelle virtù. Se il vigore dell'appetito si disperde in molti oggetti, diventa molto più debole di quello volto totalmente ad un unico oggetto. Perciò, quando l'appetito della volontà viene volto ad un altro oggetto che non sia la virtù, la volontà diventa più debole nei confronti della virtù stessa (cfr. S I $, 10,1)$.

Tra i danni degli appetiti il principale è quello di accecare e oscurare l'anima. Ogni appetito acceca e oscura l'anima, perché esso da sé stesso è cieco e non ha nessuna conoscenza. Per questo proprio l'intelletto deve essere per lui l'unica guida. 
Come i vapori oscurano l'aria e non permettono che il sole risplenda, così l'anima occupata dagli appetiti ha l'intelletto offuscato e non permette che il sole della ragione naturale o quello soprannaturale della sapienza di Dio la investano e la illuminino del tutto (Cfr. S I,8,1). L'appetito è "come il fuoco che riscalda col suo calore e abbaglia con la sua luce, nell'anima accende la concupiscenza e abbarbaglia l'intelletto di modo che non può vedere la luce" (S I,8,3). L'oscuramento e il disordine dell'intelletto fa sì che anche "la volontà è intorpidita, la memoria diventa rozza e così l'anima diviene disordinata nel suo modo naturate di operare" (S I,8,2). Qui si presenta l'unità interiore dell'uomo: la sua dimensione sensitiva e quella spirituale, che sono interdipendenti, si influenzano reciprocamente. In questo caso si manifesta l'influsso dannoso della sfera sensitiva, guidata dagli appetiti, su quella superiore e cioè spirituale che viene totalmente paralizzata nella sua attività.

Inoltre, se le potenze spirituali: intelletto, memoria e volontà sono paralizzate nel loro funzionamento, è chiaro che la persona deve essere paralizzata anche nel suo rapporto con Dio che si attua mediante la fede, la speranza e la carità che sono gli "oggetti propri soprannaturali di quelle potenze" (S II,6,1), cioè dell'intelletto, della memoria e della volontà.

Considerando i danni causati dagli appetiti e tenendo conto degli altri termini affini usati da Giovanni della Croce, come: soddisfazione, cupidigia, voglie, concupiscenza, vediamo a livello psicologico che "si tratta dell'affettività sensibile fuorviata" anche se contro l'intelletto e l'ordine morale ${ }^{12}$. Gli appetiti si presentano alla coscienza della persona come necessità o passatempi inoffensivi. Li giudica in base alla legittimità o all'indifferenza dell'oggetto, mentre il Dottore Mistico li valuta proprio sulla base dell'attaccamento affettivo, della perdita di affetto e di libertà e dei danni descritti precedentemente ${ }^{13}$.

\section{Valutazione teologica}

Il fenomeno del disordine degli appetiti, pur essendo sperimentale nella psiche umana, è anzitutto un fenomeno di natura teologica perché è legato al peccato originale che è un evento strettamente teologico. Dopo aver descritto dal punto di vista psicologico gli appetiti, possiamo dare anche una valutazione teologica che ci indicherà maggiormente la loro natura e la loro dannosità.

11 F. Ruiz Salvador, S. Giovanni della Croce. Il santo, p. 162.

12 "La metáfora del hambre y sed es frecuente en San Juan de la Cruz y en los místicos, porque ella expresa bien la urgencia el apremio, el tormento de los apetitos humanos" (V. CAPANAGA, San Juan de la Cruz, pp. 158-159).

13 F. Ruiz Salvador, S. Giovanni della Croce. Il santo, p. 163. 


\subsection{Idolatria}

Nel capitolo quarto della Salita al Monte Carmelo, parlando degli appetiti, Giovanni enuncia un principio teologico che è alla base della loro valutazione teologica. Si tratta della verità della trascendenza di Dio rispetto alle creature. Tra l'essere di Dio e il mondo creato sussiste una distanza infinita: "Tutto l'essere delle creature, paragonato con quello infinito di Dio, è niente" (S I,4,4). Il Santo per illustrare questa distanza si serve di vari simboli contrari: luce - tenebre, bellezza - deformità, gentilezza e grazia - scortesia e goffaggine, bontà - malizia, sapienza - ignoranza, libertà - schiavitù, ricchezza e gloria - povertà e miseria, ecc (cfr. S I,4,2-7). Mediante questa esemplificazione il santo Dottore vuole mostrare come tutto ciò che è onorato nel mondo, in confronto a Dio è nulla.

Sottolineare l'infinita trascendenza di Dio significa, nello stesso tempo, mettere in rilievo la sua unicita $^{14}$ : Dio in quanto Sommo ed Eterno deve essere unico e non ci può essere un altro all'infuori di Lui. L'unicità di Dio interessa il Santo in quanto affermazione pratica ed esistenziale nella vita dell'anima. Quella pratica unicità di Dio nella vita cristiana viene minacciata nel momento in cui qualche cosa è posta accanto a Dio, ciò che praticamente avviene nel caso dell'uomo dominato dagli appetiti. L'anima affezionata attraverso il suo appetito ad una cosa creata è tanto meno capace di ricevere l'unico Dio, quanto più è radicato in lei quell'appetito. La logica della spiegazione è chiara: "Due contrari non possono essere contenuti nel medesimo soggetto [... ]: l'affetto per Dio e quello per le creature sono contrari e quindi non possono essere contenuti nella stessa volontà" (S I,6,1). Nella prospettiva sangiovannea ogni appetito si presenta quindi come un vero rivale di Dio, perché, invece di Dio, diventa esso il valore unico ed esclusivo nella vita affettiva della persona.

Il meccanismo psicologico dell'uomo fa sì che

quando la volontà si affeziona ad una cosa, la stima più di tutte le altre, quantunque migliori di quella, se non le piacciono quanto la prima. Se vuole gustare dell'una e delle altre recherà necessariamente oltraggio alla più nobile; perché le pone sullo stesso piano di uguaglianza. Poiché non vi è cosa uguale a Dio, l'anima che insieme a Lui ama qualche creatura o si attacca ad essa, reca oltraggio al Signore. Se dunque è così, che cosa sarebbe se quella amasse qualche essere creato più del Creatore? (S I,5,5).

14 Cfr. F. Forestr, Le radici bibliche della "Salita al Monte Carmelo" di S. Giovanni della Croce, in: "Carmelus", 28 (1981), p. 233. 
Così la principale conseguenza teologica degli appetiti radicati nell'anima diventa in pratica un radicale rifiuto di Dio che viene trattato come una delle creature. Anzi, "colui che ama una creatura si pone sul piano di lei, scende anzi più in basso, poiché l'amore non solo rende uguale ma assoggetta l'amante alla cosa o alla persona amata" (S I,4,3). Allora per quell'uomo la creatura diventa un vero idolo che si è impossessato di lui e che egli, in pratica, adora nella sua vita al posto del vero Dio.

Si tratta qui allora di una vera idolatria, di non osservare il primo comandamento:

Io sono il Signore, tuo Dio [...]. Non avere altri dei di fronte a me! Non ti farai idolo né immagine alcuna di ciò che è lassù in cielo, né di ciò che è quaggiù sulla terra, né di ciò che è nelle acque sotto la terra. Non ti prostrerai davanti a quelle cose e non le servirai. Perché io il Signore tuo Dio sono un Dio geloso (Dt 5,6-9).

Non solo la descrizione dell'appetito corrisponde all'idolatria, ma anche i testi biblici che Giovanni della Croce cita a proposito degli appetiti presuppongono tale identità. Quando il Santo vuole trovare nella Bibbia testi riferentesi agli appetiti, sceglie spesso passi riguardanti proprio gli idoli o l'idolatria. Così, p.e., l'espressione del salmo: "siano simili ad essi [agli idoli] coloro che in essi ripongono il loro cuore" (Ps 113,8 - Vulgata) designa colui che è attaccato ad una creatura (cfr. S I,4,3). L'episodio di Giacobbe è significativo: prima di salire sul monte Betel per edificarvi un altare a Dio e offrirvi poi un sacrificio egli ordinò alla sua gente di gettare via tutti gli idoli (Gen 35,2); san Giovanni lo applica alla necessità della purificazione degli appetiti per poter offrire a Dio "un sacrificio di puro amore" (S I, 5,7). Similmente, il tentativo dei Filistei di mettere l'arca dell'alleanza nel tempio dove si trovava il loro idolo (1 Sm 5,2-5), viene paragonato a coloro che seguono il Signore ma con l'affetto attaccato ai valori mondani (cfr. S I, 5,8). Questo tipo di argomentazione Giovanni lo continua ad usare anche nel terzo libro della Salita, quando esamina diversi attaccamenti della volontà (cfr. p.e. De 32,15 in S III,19; Gio 31,27ss in S III,28,5-6; Dan 9,27 in S III,22,4; Ger 2 in S I,6 e S III, 19,7).

Anche questo fatto dell'applicazione dei diversi brani biblici riguardanti gli idoli agli appetiti ci mostra chiaramente che il nostro Autore sente vicina tale valutazione teologale.

\subsection{Antitesi dell'amore teologale}

L'uomo che adora gli idoli e in loro ripone il suo affetto, si distoglie dal vero Dio, rendendosi incapace di aprirsi alla sua amorosa comunicazione. Per il 
nostro Santo questo è anche il principale danno di natura teologale che viene causato nell'uomo dagli appetiti: "È chiaro che l'anima, per la stessa ragione per cui si affeziona ad una cosa" creata, è tanto meno capace di ricevere Dio, quanto più è radicato in lei quell'appetito (S I,61). Spiegando questa affermazione scrive:

Due contrari non possono essere contenuti nel medesimo soggetto $[\ldots]$ : l'affetto per Dio e quello per le creature sono contrari e quindi non possono essere contenuti nella stessa volontà. [...] Pertanto, come nella generazione naturale non si può introdurre una nuova forma senza espellere prima dal soggetto quella contraria, preesistente, la cui presenza è d'impedimento all'altra per l'opposizione esistente fra loro, così finché l'anima si assoggetta alle attrattive del senso non può ricevere il puro spirito di Dio (S I, 6,2).

Dato che Dio è amore e il suo Spirito è lo Spirito dell'amore, l'uomo che a causa degli appetiti non può "ricevere" Dio e il suo Spirito, non può anzitutto ricevere e vivere il suo amore. La dinamica dell'amore tra Dio e l'uomo è tale che la persona che non ha sperimentato e vissuto l'amore di Dio, non può - come conseguenza - dargli la sua risposta d'amore e del dono totale di sé. Così per Giovanni della Croce gli appetiti sono un dinamismo contrario all'amore teologale, ed in questo senso ostacolano e paralizzano la piena realizzazione dell'uomo nella comunione d'amore con Dio. Essi arrestano l'uomo e impediscono la sua piena crescita personale nell'amore, anzi lo fanno tornare indietro nel suo cammino spirituale:

A causa di questo attaccamento [le anime] non solo non progrediscono $\mathrm{ma}$, quel che è peggio, tornano indietro rifacendo a ritroso il cammino già percorso e perdendo ciò che in tanto tempo e con tanta fatica avevano guadagnato. Sappiamo già come, nella via dello spirito, chi non va avanti torna indietro e chi non cammina guadagnando cammina perdendo" (S I, 11,5).

I due dinamismi: l'amore teologale e gli appetiti, essendo a vicenda contrari, si illuminano e si chiariscono reciprocamente nella loro comprensione: Sappiamo che l'amore teologale integra e armonizza tutta la persona umana e il funzionamento delle sue facoltà (cfr. C 27,6-8; C 28,3-5.8). Al contrario, gli appetiti che invadono e dominano l'esistenza dell'uomo, producono in lui effetti totalmente contrari a quelli dell'amore: dividono, disperdono, dissipano tutti i "beni" dell'anima (cfr. S I, 10,1).

Essendo un dinamismo contrario all'amore, gli appetiti impediscono l'unione dell'uomo con Dio che si realizza proprio attraverso l'amore (cfr. S I,4,3). È quindi chiaro che "per raggiungere l'unione con Dio, [l'anima] deve essere priva di tutti gli appetiti, per minimi che siano" (S I,11, tit.). 


\section{VALUTAZIONE MORALE}

Il Dottore Mistico, quando descrive i molteplici danni causati dagli appetiti a livello psicologico e li valuta dal punto di vista teologico come impedimento per l'unione dell'amore con Dio, idolatria e antitesi della carità teologale, distingue precisamente di quali appetiti si tratta. Nei capitoli 11 e 12 del primo libro della Salita afferma che non tutti questi appetiti sono ugualmente pericolosi e dannosi ma solo quelli volontari, per i quali l'uomo è moralmente responsabile, giacché li permette consciamente e volontariamente: "Non tutti gli appetiti sono di danno all'anima allo stesso modo; parlo di appetiti volontari" (S I,11,1); si riferisce a quelli in cui la tendenza sensibile è accompagnata dalla partecipazione della volontà. Questa partecipazione può essere attiva, quando cioè la volontà stessa muove gli appetiti trovandone soddisfazione, oppure si può parlare di responsabilità passiva, quando la volontà non reprime i moti spontanei dell'appetito $^{15}$.

Gli appetiti non volontari e cioè naturali, sono quei primi moti e tentazioni a cui la volontà non partecipa "né prima né dopo" (S I,11,2) e che "in questa vita è impossibile eliminarli" (ibid.), non le impediscono di giungere all'unione con Dio e non causano altri danni. Questo avviene perché, pur trovandosi nella parte sensitiva dell'uomo, l'intelletto e la volontà possono rimanerne completamente liberi, questo accade non raramente durante l'orazione (cfr. ibid.). Anche se la persona subisce allora una certa confusione nei sensi, essi non operano in lei i danni descritti dal Santo, anzi, combattendoli, l'uomo acquista forza, pace, luce, purezza e altri beni, contrapposti a questi danni (cfr. S I,12,6).

Solo gli appetiti volontari, anche se piccoli, sono sorgente di ogni disordine morale, di tutti i peccati dell'uomo, sia mortali che veniali, o imperfezioni. Da loro l'uomo si deve liberare assolutamente per potersi unire a Dio, poiché tale unione è frutto della totale armonia della volontà umana con quella di Dio. Se, quindi l'uomo desidera qualche imperfezione, che senz'altro Dio non vuole, questa armonia non è piena (cfr. S I, 11,2-3).

In modo particolare è qui pericolosa la creazione di abitudini permanenti che sorgono dalla ripetizione di imperfezioni volontari, per esempio:

l'abitudine comune di parlar molto, un piccolo attaccamento a qualcosa che l'anima non si decide mai a superare, che sia una persona, un vestito, un libro, una cella, un determinato cibo, futili conversazioni, piccole voglie sia verso cose, sia di sapere, di udire e simili $($ S I,11,4).

15 Cfr. F. Ruiz Salvador, S. Giovanni della Croce. Il santo, p. 664. 
Se l'attaccamento diventa ossessivo, ha poca importanza che i suoi oggetti siano delle inezie. Ognuna di queste imperfezioni, cui si è legati e abituati, perfino la più piccola, rende impossibile il progresso morale e spirituale perché come nota san Giovanni - "non ha importanza che sia sottile o grosso il filo con cui è legato un uccello, perché questo rimarrà prigioniero, sia nell'uno che nell'altro caso, fino a quando non l'avrà spezzato" (S I,11,4).

È da notare che quest'abitudine, per il fatto che nella sua creazione partecipa la volontà, ha anche un carattere morale, per questo pare che non sia giusto cercare troppi parallelismi tra essa e il concetto di fissazione di cui parla la psicoanalisi, che si sono fatti in riferimento proprio a questo capitolo 11 del secondo libro della Salita per giustificare la tale affermazione ${ }^{16}$. Infatti, la fissazione psicoanalitica, contrariamente a ciò che è descritto da Giovanni della Croce come fenomeno, è più di carattere inconscio in cui non partecipano la responsabilità e la volontà umana ${ }^{17}$.

Ogni appetito volontario produce nell'anima tutti i mali che si sperimentano a livello psicologico, cioè la stanca, la tormenta, l'oscura, l'insudicia e l'indebolisce, operando in modo contrario ad ogni virtù che, per natura, genera nella psiche umana gli effetti positivi (cfr. S I, 12,5). Come con l'esercizio di una virtù crescono tutte le altre, così è per i vizi e $i$ danni da essi operati che aumentano quando aumenta uno solo di loro. Di solito questi danni non si possono vedere tutti nel momento in cui l'appetito viene soddisfatto, perché il piacere che si prova allora non lo permette, tuttavia prima o poi se ne sperimentano le funeste conseguenze (cfr. S I, 12,5).

L'appetito, come tendenza disordinata, contraria all'amore teologale, sta a fondamento di ogni peccato. Si può dire che per mezzo degli appetiti il peccato - in una certa maniera - "è presente, lavora e distrugge in modo continuo, pure nei momenti in cui non si traduce in atti” ${ }^{\prime 1}$. Possiamo però parlare di peccato in senso proprio e di responsabilità morale quando lasciamo che gli appetiti sorgano volontariamente in noi.

16 Cfr. F. Urbina, Comentario a Noche oscura del espíritu y Subida al Monte Carmelo de san Juan de la Cruz, Madrid 1982, pp. 33-37.

17 Cfr. W. Toman, voce: Fissazione, in: Dizionario di psicologia, a cura di W. Arnold, H.J Eysenck, R. Meili, Milano 1986, p. 425. Su questo tema F. Ruiz dà la seguente valutazione: "Si è parlato... di un certo parallelismo esistente tra appetito sangiovinneo e fissazione psicoanalitica. L'insistere del Santo su termini come bambini e infantilità, attaccamento e abitudine, così come sull'effetto paralizzante nei confronti del dinamismo affettivo, creerebbe una certa affinità tra appetito e fissazione.

Penso tuttavia che, anche senza uscire dal piano psicologico, le divergenze siano molto più serie dei punti di contatto. L'appetito di cui parla san Giovanni della Croce comporta, nei confronti della fissazione, maggiore partecipazione della coscienza, della volontà, della responsabilità vocazionale" (F. Ruiz Salvador, S. Giovanni della Croce. Il santo, p. 164).

18 F. Ruiz Salvador, Purificazione affettiva e lotta contro il peccato, in: Peccato e santità, Roma 1979 , p. 146. 


\section{Prime CURE PER LA PURIFICAZIONe Degli APPETITI}

La necessità della purificazione degli appetiti dalla loro sregolatezza nasce dalla loro valutazione teologale e morale e dai danni che causano sia a livello psicologico che spirituale e morale.

La base teologica della necessità della purificazione degli appetiti intesi come idolatria, è il primo comandamento del Decalogo. Esso, espresso nel Dt 5,6-9 in forma negativa: "Non avere altri dei di fronte a me!..." riceve la sua formulazione positiva nel precetto dell'amore totale: "Tu amerai il Signore tuo Dio con tutto il cuore, con tutta l'anima e con tutte le forze" (Dt 6,5). Secondo la dinamica dell'amore è l'amore stesso che spinge e dà la forza necessaria per la purificazione: l'amore comandato, ma accolto prima da Dio come dono.

Dt 6,5 rimane un riferimento basilare per la purificazione degli appetiti sensitivi, anche se è espresso da Giovanni della Croce implicitamente quando, con uno sguardo retrospettivo, riassume la notte dei sensi; per entrare in essa l'anima "deve restringere e spogliare la propria volontà di tutte le cose sensibili e temporali, amando Dio al di sopra di tutto. Questo lavoro appartiene alla notte del senso, di cui ho già parlato" (S II, 7,2). Proprio quest'amore di Dio "al di sopra di tutto" che si concretizza nell'amore ansioso e appassionato di CristoSposo, sta alla base della purificazione degli appetiti, come forza che permette all'anima di rinunciare al richiamo seducente dell'amore sensibile:

Era $[\ldots]$ necessaria all'anima la fiamma più potente di un amore maggiore cioè di quello del suo Sposo, perché ella, riponendo in Lui il proprio gusto e la propria forza, avesse il coraggio e la costanza di rinnegare senza difficoltà tutti gli altri piaceri. Tuttavia per vincere la forza degli appetiti sensitivi non basta che l'anima ami semplicemente il suo Sposo, ma si richiede che arda di amore ansioso per lui (S I,14,2).

Ogni sforzo dell'uomo, teso a purificare il disordine degli appetiti, affinché sia efficace, deve discendere da quell'amore di Cristo che ha ricevuto da Lui ed ha sperimentato nella propria vita. Esso è una virtù dalla quale nasce nell'uomo il desiderio di purificarsi e tale opera si compie per mezzo di essa. Nei capitoli 13-15 del primo lñibro della Salita al Monte Carmelo e nel capitolo 7 del secondo libro, il nostro Autore dà i principi generali, le prime cure pratiche che devono essere attivamente intraprese dall'uomo, mosso dall'amore, per purificarsi dagli appetiti ${ }^{19}$.

19 "Gli avvisi che ora darò per vincere gli appetiti, quantunque pochi e schematici, sono, a mio parere, tanto utili ed efficaci quanto concisi; colui, quindi, che li metterà in pratica non avrà bisogno di altri perché questi li compendiano tutti" (S I, 13,2). 


\subsection{Imitazione di Gesù}

L'amore di Cristo, se è vero, porta sempre al desiderio di imitarlo. Questo è anche il primo e il più importante avviso dato da san Giovanni per la purificazione degli appetiti: "In primo luogo, l'anima abbia un costante desiderio di imitare Cristo in ogni sua azione, conformandosi ai suoi esempi, sui quali mediti per saperli imitare e per comportarsi in ogni sua azione come Egli si deporterebbe" (S I, 13,3).

L'amore di Cristo viene qui espresso come un "costante desiderio" (ordinario apetito) per sottolineare che solamente esso può combattere l'appetito abituale alle creature ${ }^{20}$. Quest'amore deve esprimersi in pratica nella sequela di Gesù, ciò presuppone un processo di adesione incondizionata alla Persona del Signore e al suo messaggio, entrando pienamente nell'orbita della sua influenza e lasciandosi costantemente guidare da Lui. La Parola di Dio e la preghiera ci offrono un grande aiuto nella scoperta e nell'adesione a Cristo. È la Parola di Dio che ci presenta la vita storica di Gesù ed il suo mistero, la preghiera è invece un luogo privilegiato per la meditazione della sua vita a cui invita Giovanni per poterlo poi imitare "in ogni sua azione, conformandosi ai suoi esempi". Ciò significa, in pratica, personalizzare la vita di Gesù, i suoi atteggiamenti, un continuo domandarsi come Egli si sarebbe comportato nelle diverse circostanze della vita ordinaria se fosse stato al mio posto.

Poiché la Pasqua di Gesù è il punto centrale della sua vita e della sua missione, tale evento deve essere meditato intensamente. La Sua passione e morte, che si esprime nella figliale obbedienza al Padre, ed il suo amore per gli uomini fino al totale annientamento fisico e spirituale ed alla piena negazione di sé, diventa per Giovanni oggetto di una profonda meditazione (cfr. S II,7). Vi è una pagina veramente significativa che merita di essere riportata per intero:

[...] Cristo è la via e questa consiste nel morire alla natura in ciò che appartiene al senso e allo spirito [...] In primo luogo è certo che Gesù morì, spiritualmente in vita e naturalmente in morte, a tutto ciò che cade sotto il dominio dei sensi, poiché come Egli stesso disse, in vita non ebbe un luogo dove reclinare il capo (Mt 27,44) e tanto meno lo ebbe in morte. In secondo luogo è evidente come, al momento della morte, Egli fosse annichilato anche nell'anima, senza alcun sollievo e conforto, essendo stato lasciato dal Padre secondo la parte inferiore, in un'intima aridità, così grande che fu costretto a gridare: "Dio mio, Dio mio, perché mi hai abbandonato?" (Mt 27,44). Quello fu l'abbandono più desolante che avesse sperimentato nei sensi durante la sua vita e, proprio mentre ne era oppresso, Egli compì l'opera più

20 Cfr. F. Ruiz Salvador, S. Giovanni della Croce. Il santo, p. 165. 
meravigliosa di quante ne avesse compiute in cielo e in terra durante la sua esistenza terrena ricca di miracoli e di prodigi, opera che consiste nell' aver riconciliato e unito a Dio, per grazia, il genere umano. [...]

Il Signore ha compiuto ciò perché la persona spirituale, per unirsi con Dio, intenda il mistero della porta e della via di Cristo e sappia che quanto più ella si annienterà per Dio, secondo la parte sensitiva e quella spirituale, tanto maggiore unione con Lui raggiungerà e tanto maggiore sarà la sua opera. [...] La via di cui parla Nostro Signore non consiste in diletti, gusti e sentimenti spirituali, ma in una vera morte di croce sensitiva e spirituale, cioè esteriore e interiore (S II,7,9-11).

Giovanni della Croce sottolinea l'annientamento totale di Gesù nella sua Passione e morte, e ricordando, proprio in questo contesto, che Egli "è la via", mostra che è anche la via per la piena unione con Dio, quindi, anche per la purificazione sia dei sensi che dello spirito, senza la quale l'unione non è possibile. Bisogna però notare che l'annientamento di Cristo in croce non è che un aspetto della Passione ${ }^{21}$. Nella morte di Gesù si ha la rivelazione ultima di Dio, il dono supremo del suo amore: nella morte di Gesù si manifesta la perfezione della carità. Di per sé, non sono l'annientamento e la morte di Gesù che salvano il mondo, ma il suo amore.

Anche per il Dottore Mistico l'annientamento purificatore dell'uomo sull'esempio di Cristo - dev'essere opera dell'amore, altrimenti sarà soltanto un cammino di morte: "nella sua solitudine eterna, anche il dannato vive un suo annientamento" ${ }^{22}$. L'esigenza dell'imitazione amorosa di Gesù, specialmente della sua Passione, per l'importanza che il Santo le attribuiva, era un punto che ricorreva nel suo insegnamento orale. Tale espressione si ritrova negli avvisi e nelle sentenze che rivolgeva a diverse persone, p.e.: "Le basti Cristo Crocifisso, con Lui soffra e riposi e perciò si renda un nulla in tutte le cose esteriori ed interiori" (SA 13). "Abbia in cuore la forza contro tutte le cose che vorrebbero condurla a ciò che non è Dio e sia amica della Passione di Cristo" (SA 16). "Se vuoi esser perfetto, vendi la tua volontà e dalla ai poveri di spirito, vieni a Cristo nella mansuetudine ed umiltà e seguilo fino al Calvario e al sepolcro" (AA 6).

21 Specialmente nel Vangelo di Giovanni si vede l'insistenza prima di tutto sull'amore e sulla libertà sovrana di Gesù nella sua Passione (cfr. Gv 3,16;10,17-18; 16,28; 17,19).

22 D. BarsotTr, La teologia spirituale di san Giovanni della Croce, Milano 1990, p. 48. 


\subsection{Rinuncia e mortificazione abituale in tutto}

Per imitare effettivamente Gesù e perseverare in Lui, viene dato un altro suggerimento concreto: "In secondo luogo, per riuscire in questo è necessario che [l'anima] rinunci a qualunque piacere sensibile che non sia puramente a onore e gloria di Dio e che rimanga vuota di ciò per amore di Gesù Cristo" (S I,13,4).

Si tratta, quindi, di concentrare la propria attività solo su ciò che è la volontà di Dio evitando ogni evasione e compensazione, rinunciando quindi a tutto ciò che disperde per vivere coscientemente questo vuoto di gratificazioni, riposando nella sufficienza di Dio ${ }^{23}$. Come criterio teologico e misura di questa rinuncia al piacere sensibile il santo Dottore indica "l'onore e la gloria di Dio" che concretamente significa vivere la propria vocazione $e^{24}$. Quindi, in pratica, il significato di criterio teologico considerato precedentemente, consisterebbe nell'evitare cose e sensazioni ricercate per soddisfazione o passione, che non siano in armonia con la propria vocazione. La motivazione e la forza di quest'opera deve essere naturalmente, "per amore di Gesù Cristo".

Non si tratta di rinuncia e mortificazione qualsiasi, devono essere perfette e prese con determinazione e coraggio, affinché possano diventare un mezzo efficace per purificare gli appetiti sregolati ${ }^{25}$. In concreto questa rinuncia e mortificazione consistono in che "il vero spirito cerca nel Signore più l'amaro che il dolce, propende più per le sofferenze che per le consolazioni, si sente spinto per amore di Dio più alla rinuncia che al possesso di ogni bene, tende più alle aridità e alle afflizioni che alle dolci comunicazioni, sapendo bene che solo così si segue Cristo e si rinuncia a se stessi e che agire altrimenti vuol dire cercare se stessi in Dio, cosa molto contraria all'amore" ( $\mathrm{bid}$.). Lo stesso programma, espresso ancora più dettagliatamente, si trova nel capitolo 13 del secondo libro della Salita al Monte Carmelo

Per mortificare e calmare le quattro passioni naturali $[\ldots]$ l'anima cerchi sempre di inclinarsi: non al più facile, ma al più difficile; non al più saporoso ma al più insipido; non a quello che piace di più, ma a quello che piace di meno; non al riposo, ma alla fatica; non al conforto, ma a quello che non è conforto; non al più, ma al meno; non al più alto e pregiato, ma al più vile e disprezzato; non alla ricerca di qualche cosa, ma a non desiderare niente;

Cfr. J. Garrido, Relectura de San Juan de la Cruz, Madrid 1991, p. 92.

Cfr. F. Ruz Salvador, S. Giovanni della Croce. Il santo, p. 165.

"Alcune [persone] sono convinte che basti qualunque genere di ritiro e di riforma della vita, altre si contentano di praticare in qualche modo le virtù, di dedicarsi all'orazione e di esercitarsi nella mortificazione, ma né le une né le altre raggiungono la nuda povertà, l'abnegazione o la purezza spirituale, che sono tutt'uno, consigliate da Nostro Signore. Esse, infatti, si preoccupano più di nutrire e di vestire la loro natura di consolazioni e di sentimenti spirituali che di spogliarla e privarla per amore di Dio da ogni cosa" (S II,7,5). 
non alla ricerca del lato migliore delle cose create, ma del peggiore e a desiderare nudità, privazioni e povertà di quanto v'è al mondo per amore di Gesù Cristo (S I, 13,6).

La finalità di queste norme che sembrano così paradossali, è quella di riconquistare la libertà della carità di fronte alle passioni, alle comodità, all'egoismo. Di per sé il difficile non deve necessariamente valere di più. Per la persona in cui l'amor proprio è presente e spesso si camuffa di tanti artifici, l'unico modo di non lasciarsi ingannare è il rigore assoluto: "sono in generale più preziose e accette davanti a Dio le azioni in cui l'uomo maggiormente si mortifica, specialmente quando uno non è molto innanzi nella perfezione, a causa della rinuncia a sé stesso che vi porta, che quelle in cui egli trova conforto, perché è più facile in queste cercare se stesso" (S III,28,8).

Come rimedio efficace l'Autore suggerisce questo sistema che rovescia l'ordine naturale delle preferenze scegliendo ciò che è difficile, insipido, faticoso e disprezzato però sempre nella prospettiva teologale: per amore di Gesù Cristo. Questo esercizio ha già in sé il valore dell'amore sacrificato e della carità fraterna lasciando a loro ciò che è più facile e piacevole ${ }^{26}$.

\section{3. "Nada" per "todo"}

La rinuncia e la mortificazione di cui parla il Santo provengono sempre dall'amore e sono in vista ad un amore più grande. Nella dinamica dell'amore è la persona intera che, conosciuto l'amore di Dio, giunge all'unione con Lui. Per farlo in modo indiviso: "con tutto il cuore, con tutta l'anima, con tutte le forze" (Dt 6,5) essa non guarda alle cose che lascia concentrando le sue forze nell'Amato e nel bene che spera di ricevere. La sua prima intenzione è quella d'incontrare Dio. Se, per raggiungere tale fine, dovesse fare la rinuncia più dolorosa, non sentirebbe alcun dolore, purché questa serva effettivamente al fine propostosi.

Giovanni della Croce consapevole di questa verità ha scritto sul portale della Salita al Monte Carmelo, ai piedi del monticello della perfezione le parole programmatiche di questa logica dell'amore:

Per giungere a gustare il tutto,

non cercare il gusto in niente.

Per giungere alla conoscenza del tutto,

non cercare di sapere qualche cosa in niente.

Per giungere al possesso del tutto,

26 Cfr. F. Ruiz Salvador, S. Giovanni della Croce. Il santo, p. 167. 
non voler possedere niente.

Per giungere ad essere tutto, non voler essere niente ${ }^{27}$.

Come un ritornello si ripete il binomio "tutto" (todo) - "niente" (nada). "Tutto" e "niente" per il Santo di Fontiveros non sono categorie filosofiche del dualismo platonico, ma categorie interpersonali, poiché esprimono la verità sulla relazione tra Dio e l'uomo. Il "tutto" non è "qualcosa", è "Qualcuno": "Il Padre ci donò tutto, cioè suo Figlio" (S II 22,4 ). Questa è la definizione del "tutto" offerta dal Santo e mostra che questo "Tutto" - Cristo è per noi. Lo gustiamo, lo conosciamo e lo possediamo solo quando impegnamo tutto il nostro essere, in particolare le facoltà spirituali, ad aderire a Lui tramite le virtù teologali, a cui il testo fa riferimento ${ }^{28}$.

Ogni altro mezzo utilizzato senza questo riferimento teologale a Cristo è quindi "niente" cioè non conduce a tale scopo. Ogni attaccamento disordinato ed eccessivo a qualcosa creata ci priva del "Tutto", perché "tutto l'essere delle creature, paragonato con quello infinito di Dio, è niente" (S I,4,4). Così anche l'uomo in forza di questo attaccamento diventa "niente" perché "l'amore ha la proprietà non solo di rendere simili ed uguali, ma di collocare chi ama in condizione di inferiorità rispetto all'amato" (ibid.). Questo lo fa il dinamismo dell'amore.

Il radicalismo compreso nel programma "tutto" - "niente" se viene guardato "dal di fuori", senza esperienza radicale d'amore, suona inumano e solo metafisico. Se si percepisce però "dal di dentro", qual è la forza interiore che lo muove, si nota come tutta l'ascesi si concentra ultimamente nell'amore e ciò semplifica molto la visione del cammino spirituale. La pedagogia di San Giovanni racchiusa nel cammino di negazione mira al nucleo della purificazione che consiste nell'amare Dio solo, distaccandosi da tutto ${ }^{29}$. Colui che segue questo cammino ha trovato la scorciatoia nella salita al Monte e cammina senza aggiramenti tortuosi.

27 Citiamo qui alcune frasi che sembrano più rilevanti. Sono state ripetute anche nella S I, 13,11 .

28 "C'è una corrispondenza fra potenze e virtù teologali: volontà - carità (gustare), intelletto fede (sapere), memoria - speranza (possedere)" (F. Runz Salvador, S. Giovanni della Croce. Il santo, p. 492).

29 Cfr. J. Garrido, Relectura de San Juan de la Cruz, p. 95. 


\section{Conclusione}

Il tema degli appetiti è senza dubbio uno dei più importanti per Giovanni della Croce. Esso è strettamente legato - da una parte - alla natura umana, creata da Dio come natura "affettiva", però - dall'altra parte - anche alla realtà del peccato e delle sue conseguenze nella vita dell'uomo. La scelta di rifiutare il progetto di Dio verso l'uomo e di vivere la vita secondo le proprie idee ha causato che l'esistenza umana non sia più vissuta con senso teologale. In conseguenza l'uomo rompe ugualmente la sua armonia interiore e comincia a vivere sotto il segno della contraddizione, della rottura e del conflitto causato dagli appetiti e dalle passioni che a causa di questa scelta diventano disordinati. Così, poco a poco, si comincia a vivere senza riferimento trascendente, un'esistenza dove Dio diventa sempre più estraneo e dove la realtà creata è vissuta con riferimento ad un "io" egoistico, possessivo e chiuso nei propri limitati orizzonti ${ }^{30}$.

Partendo da questa visione generale, basandosi sulla Salita al Monte Carmelo -è stato esaminato il significato e il ruolo degli appetiti. Nella concezione filosofica dell'uomo il nostro Santo è ancorato al mondo filosofico del tomismo, tuttavia non è legato né segue pedissequamente alcun sistema o alcun autore. Così anche gli appetiti non vengono analizzati da lui astrattamente, ma nel concreto della vita e di conseguenza vengono presentati come l'ostacolo principale che sbarra la strada dell'unione con Dio. La loro descrizione, anche se da un punto di vista sistematico non è accurata e presenta delle lacune (p.e. manca la loro precisa definizione), da un punto di vista psicologico, teologico e morale è quanto mai abbondante, ricca e suggestiva. Si parla molto dei danni causati in ognuno di questi livelli e - indirettamente - dei vantaggi a loro contrari. Si vede inoltre una grande unità dei diversi aspetti sotto i quali il Santo analizza gli appetiti.

Anzitutto egli scopre, nei fenomeni e nel comportamento umano, intime relazioni tra l'elemento psicologico e quello morale. Così gli appetiti a cui l'uomo soccombe consapevolmente e volontariamente (aspetto morale) gli causano gravi danni a livello psichico (aspetto psicologico). Al contrario, per arrivare alla purezza e rettitudine che formano l'aspetto morale, occorre la rinuncia, la mortificazione, lo spogliamento della volontà che si sperimenta molto a livello psicologico. Questo si vede ancora di più nella purificazione passiva, dove le imperfezioni morali vengono tolte con la medicina dell'aridità, del vuoto e dell'oscurità presenti nella psiche umana.

La ragione di quest'unità e connessione si trova nel fatto che Giovanni della Croce, per dare una classificazione dei fenomeni psichici, non parte dal piano psicologico, né, per analizzare la dimensione morale del comportamento, par-

30 Cf. A. Baldeón, El hombre: una pasión de amor. Comprender la vida desde San Juan de la Cruz, Burgos 1991, p. 119. 
te dal piano morale, ma va più in profondità fino ad arrivare al piano teologico, dal quale, del resto, comincia a trattare questo tema ${ }^{31}$. Qui scopre le strutture essenziali manifestate dai fenomeni psicologici e dalle quali derivano anche gli atteggiamenti morali. Questa realtà teologica fondamentale è Dio e l'uomo che si "cercano" reciprocamente per incontrarsi: Dio nella sua trascendenza e l'uomo nella sua miseria ${ }^{32}$. L'esperienza della grazia di tale "ricerca" dell'uomo da parte di Dio, attuata in Cristo, diventa per l'uomo una forte spinta per imitare Gesù e per intraprendere le prime cure per purificare gli appetiti, allo scopo di conquistare la direzione della propria vita per porla al servizio di Cristo.

È anche la grazia attuata nelle virtù teologali che, con la docilità e collaborazione dell'uomo, gli dà una tensione radicale verso Dio e lo unisce con Lui pienamente dopo il processo di purificazione passiva, operando inoltre la sua completa integrazione ai livelli più profondi dell'essere, conducendolo alla propria pienezza, possibile in questa vita, e aprendolo agli orizzonti escatologici.

31 Cf. S I, 4,1-6,4. Soltanto dopo tratta del piano psicologico (S I, 6,5-10,4) e di quello morale (S I,11-12) degli appetiti.

32 "L'uomo è segnato da Dio dal duplice abisso della sua finitezza e del suo peccato: Dio è alto, prodigioso, santo; l'uomo è basso, corto, vile e peccatore. Finché Dio e l'uomo restano ciò che sono, non si possono unire. Per questo, tutto ciò che appartiene all'ordine dell'impurità - peccato e conseguenze del peccato, tutto ciò che appartiene all'ordine della bassezza - maniera umana di pensare, di amare e di agire, tutto ciò deve essere radicalmente distrutto, affinché l'anima sia cambiata da 'vecchia' in 'nuova', trasformata e letteralmente divinizzata" (J. Mouroux, Nota sull' affettività sensibile in san Giovanni della Croce, in L'esperienza cristiana, Brescia 1956, pp. 304-305). 


\section{ABSTRACT \\ Andrzej Ruszata OCD \\ Appetites as Lost Energies according to the "Ascent of Mount Carmel" of St John of the Cross}

The theme of appetites is without doubt one of the most important for John of the Cross. It is closely linked to the reality of original sin and its consequences in the life of man. After having rejected the plan of God, man has chosen to live alone and in this rebellion, consists of his sin. It, breaking the orientation toward God of human existence that is no longer living with the theological sense, breaks equally inner harmony of man that begins to live under the sign of contradiction, of rupture and the conflict caused by the appetites and passions disordered.

Starting from this general vision based on the Ascent of Mount Carmel - in the article has been examined the significance and role of appetites. In the philosophical conception of man St John of the Cross is anchored to the world of philosophy of Thomism, however he is not bound nor (he) follows blindly any system or any author. So even appetites are not analyzed by him in the abstract but in concrete life and consequently are presented as the main obstacle that bar the path of union with God. Their description, even if from the point of view of systematic is not accurate and has certain shortcomings (e.g. missing their very precise definition), from a psychological point of view, theological and moral is whatever abundant, rich and evocative.

First of all, he discovers in the phenomena and in human behavior intimate relations between the psychological element and the moral. Thus, appetites to which man succumbs knowingly and willingly (moral aspect) the cause serious damage to psychic level (psychological aspect). On the contrary, to arrive to the purity and uprightness that form the moral aspect, must be the renunciation, mortification, emptying of the desire that you experience very on a psychological level.

The reason for this unit and connection lies in the fact that John of the Cross, to give a classification of psychic phenomena, don't start from a psychological level, nor, to analyze the moral dimension of the behavior, don't part from the moral level, but goes deeper until you arrive at the theological level, from which the rest begins to deal with this topic. Here he discovers the essential structure expressed by psychological phenomena and from which it is derived also the moral attitudes. This theological reality essential is God and man that "search" reciprocally to meet: God in his transcendence and man in his 
misery. The experience of the grace of such a "quest" of man by God performed in Christ becomes for man a strong thrust to imitate Jesus and to undertake the first treatments for purifying appetites, in order to win the leadership of his own life to put it at the service of Christ.

It is also the grace implemented in the theological virtues, witch - with the docility and collaboration of man - gives him a radical voltage toward God and unites with Him fully after the purification process passive, making its complete integration to the deeper levels of being, leading it to its fullness, possible in this life, and opening him to the eschatological horizons.

Słowa klucze: św. Jan od Krzyża, pożądania, oczyszczenie, umartwienie, wyrzeczenie, miłość

Keywords: St John of the Cross, appetites, purification, mortification, renunciation, love 